\title{
CONOCIMIENTOS, ACTITUDES Y PRÁCTICAS DE LOS DONANTES DE SANGRE EN EL MUNICIPIO DE MOCOA, PUTUMAYO EN EL AÑO 2008
}

Knowledge, attitudes and practices regarding voluntary blood donation to the inhabitants of the town of Mocoa, Putumayo in 2008

Ana Lucya Legarda Ceballos. Especialista en Epidemiología, Universidad Surcolombiana, Neiva.

Correspondencia: e-mail: chaquilula@hotmail.com

\section{RESUMEN}

Objetivo: Determinar los conocimientos, actitudes y prácticas con relación a la donación voluntaria de sangre de los habitantes del municipio de Mocoa de Marzo-Mayo de 2008.

\section{METODOLOGÍA}

Cuantitativa. Se investigó en el municipio de Mocoa, los conocimientos, actitudes y prácticas de la población de donantes con respecto a la sangre, donación y transfusión. Esto se logró con ayuda de técnicas de entrevista semiestructurada, basada en la aplicación de encuestas, donde se identificaron elementos útiles para formular una propuesta integral que promueva la cultura de la donación voluntaria o altruista no remunerada.

\section{RESULTADOS}

Se identificaron los conocimientos de las personas sobre la donación, actitudes y prácticas, y también algunas barreras y motivaciones.

\section{CONCLUSIÓN}

Los conocimientos son insuficientes porque quienes handonadoenalgúnmomento, tienen unconocimiento general sobre la sangre, la donación y en menor grado sobre la transfusión. Quienes lo hicieron por primera vez tienen creencias erróneas. Así mismo algunos donantes por reposición, manifiestan que debería existir una retribución. Además la información servirá como base, para diseñar una estrategia en la región, encaminada a promover la captación y lealtad de los donantes voluntarios de sangre, cambios necesarios en la atención y motivación para los donantes regresar con cierta regularidad a donar sangre.

\section{PALABRAS CLAVE}

Donación de sangre, transfusión.

\section{ABSTRACT}

Objective:

To determine knowledge, attitudes and practices regarding voluntary blood donation on the part of the inhabitants of the municipality of Mocoa, from March to May, 2008.

Methodology:

Quantitative. It was investigated the population of donors' knowledge, attitudes and practices with regard to blood donation and blood transfusion. 
This was achieved with the help of techniques such as semi-structured interview based on the conduction of surveys. Useful elements were identified which were used to formulate a comprehensive proposal in order to promote the culture of voluntary donation.

\section{Results:}

It was identified people's knowledge about donation, attitudes and practices, as well as some barriers and motivations.

\section{Conclusion:}

Blood donors showed insufficient knowledge. They just have a general knowledge about blood donation and to a lesser degree about transfusion. First-time donors have mistaken beliefs. Likewise for some replacement donors there should be a reward. Furthermore, based on that information it will be designed a strategy in the region seeking to promote the recruitment and loyalty of voluntary blood donors. These changes are necessary to improve service and increase donors' motivation towards more often blood donation.

\section{KEY WORDS}

Donation of blood, transfusion

\section{INTRODUCCIÓN}

Las transfusiones de sangre y de sus componentes, constituyen el tratamiento más utilizado para compensar las pérdidas de sangre y las anemias. En todos los casos, la unidad de sangre donada por una persona (el donante), es la que hace posible la transfusión sanguínea. El donante es el primer eslabón que determina la eficiencia de este proceso, sujeto a controversias, debido a los efectos adversos que pueden estar asociados con él, pero vital, ya que aún no ha podido ser reemplazado por ningún otro tipo de terapia. Con el fin de prevenir los riesgos asociados a la transfusión, se han tomado distintas medidas, entre ellas la aplicación de criterios para la selección de los donantes, la aplicación de pruebas de tamizaje y la inactivación de virus. La selección de la población de donantes, es la medida que ha tenido mayor impacto sobre la seguridad de la sangre. Se ha demostrado que una alta población de donantes voluntarios, está asociada con una baja tasa de transmisión de los agentes infecciosos que suelen vincularse con la transfusión1.

La donación de sangre, consiste en extraer una cantidad de 400 a $500 \mathrm{~mL}$ de esta, directamente de la vena del donante, a una bolsa múltiple de plástico que contiene anticoagulante y soluciones nutrientes. Los donantes tienen que realizar primero una pequeña entrevista para descartar problemas graves de salud, sobre todo que exista alguna posibilidad de contagio. Se trata de evitar enfermedades infecciosas como la hepatitis, la sífilis o el sida; después con un pequeño pinchazo se descarta que el donante tenga anemia y luego se procede a la extracción de una unidad, es decir $450 \mathrm{~cm} 3$ de sangre, luego confirmar que la sangre donada esté libre de infecciones y pueda ser utilizada sin complicaciones. Los hombres pueden donar cuatro veces al año y las mujeres tres, debido a que suelen tener más problemas de insuficiencia ferrosa. Las necesidades de sangre están creciendo ante los avances de la medicina ${ }^{2}$.

La donación de sangre en Colombia, es un acto del cual se han creado mitos y supersticiones como engordar o adelgazar, disminución de la capacidad sexual, la posible transmisión de enfermedades y la concepción de posibles infecciones, que se pueden adquirir por donar sangre, esto debido al desconocimiento en los métodos de extracción y procesamiento de la sangre; además no existe la cultura de la donación altruista voluntaria, la mayoría de las donaciones son dirigidas o por reposición con implicaciones económicas y sociales de trascendencia ${ }^{3}$.

En el municipio de Mocoa, ubicado en el departamento del Putumayo, la entidad encargada de captar donantes, es la Empresa Social de Estado (ESE), Hospital José María Hernández, quien brinda este servicio desde 1.992, siendo el único en el departamento, que procesa la sangre captada en los componentes que la conforman. Durante este tiempo se ha caracterizado por ser un banco de sangre que capta donantes, distribuye sangre y hemocomponentes sanguíneos, a los servicios transfusionales del departamento y, apoya a la vigilancia epidemiológica de la región, pero carece de información precisa y pertinente sobre aspectos socioculturales de la población, que permita establecer las causas de la baja participación en los programas de donación de sangre. 
Se puede manejar y educar en la cultura de la donación altruista voluntaria, a los habitantes de Mocoa, los cuales serán una valiosa fuente de difusión para que pronto emprendan dicha práctica, disminuyendo los índices de transmisión de enfermedades infecciosas, a través de la donación de sangre y haciendo de la terapia transfusional, un medio seguro y eficaz de tratamiento.

Hasta el momento no se han realizado investigaciones sociales en el municipio, que aborden el tema de la donación, las características socioculturales de los donantes y no donante de sangre, sus sentimientos, motivaciones, conductas y actitudes en torno a la donación y la transfusión. Con este trabajo se buscara estar al tanto de los conocimientos, actitudes y prácticas de los habitantes de Mocoa, respecto al tema, el cual será la base de posteriores trabajos, con el fin de educar con relación a la donación de sangre y lograr así donantes voluntarios sanos.

\section{MATERIALES Y MÉTODOS}

\section{POBLACIÓN}

El estudio es de tipo descriptivo, porque dirá las características de la población, en cuanto a la situación actual del los habitantes del municipio, respecto a la participación en la donación de sangre y se tomará dichos datos en un determinado periodo de la investigación, por lo tanto, se dice que la investigación es un estudio de corte transversal. Fue desarrollado entre marzo y mayo de 2008 en el municipio de Mocoa. La investigación tiene un enfoque cuantitativo, es un tipo de metodología que ofrece técnicas para obtener una comprensión profunda de la población en relación a un determinado tema, además se aplica mejor en la búsqueda de resultados del problema planteado y facilita el uso de instrumentos para su verificación, basado en el problema y los objetivos de la investigación. Está orientada a los resultados. Se desarrolla más directamente en la tarea de verificar y comprobar, teorías por medio de estudios muéstrales representativos, aplicando las encuestas, donde se recopilará la información, que contemplará las características de la población apta para donar.

\section{SELECCIÓN DE LA MUESTRA}

La población objeto de estudio, es de 24.050 personas, agrupadas en cuatro conglomerados que lo conforma las zonas: oriente, occidente, norte y sur del municipio (Tabla 1).
Tabla 1. Población objeto de estudio de las zonas oriente, occidente, norte y sur del municipio

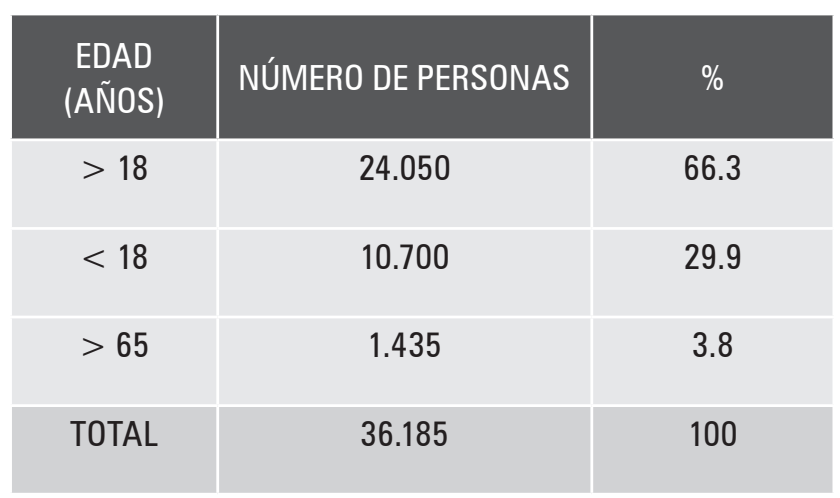

Fuente: DANE Censo 2005

Según la OMS y la OPS, la población apta para donar sangre, son las personas entre 18 y 65 años de edad, por lo tanto en Mocoa, la población apta para donar es de 24.050 personas.

\section{MÉTODOS PARA OBTENER LA INFORMACIÓN}

A fin de validar los datos y evitar sesgos, se utilizó la técnica de entrevista individual semiestructurada, basada en la aplicación de la encuesta. Este tecnica, permite determinar lo que piensan, conocen, creen, perciben y hacen las personas con respecto a la sangre, la donación y la transfusión.

\section{INSTRUMENTO}

\section{ENCUESTA}

Para la recolección de los datos, se aplicó la encuesta; un método y diseño observacional, que se usa para obtener información de una muestra de individuos. Esta "muestra" es usualmente sólo una fracción de la población bajo estudio. El tamaño de muestra requerido en una encuesta, depende en parte de la calidad estadística necesaria para establecer los hallazgos 4; esto a su vez, está relacionado en cómo esos hallazgos serán usados, que permitió la recolección de la información sobre conocimientos, actitudes, y prácticas con relación a la donación voluntaria de sangre en el municipio de Mocoa. Las escalas empleadas para medir las variables, fueron de tipo nominal y ordinal. 


\section{VALIDACIÓN DEL CUESTIONARIO}

El cuestionario usado en este estudio, se sometió a prueba con de 16 personas representantes de cada uno de los conglomerados definidos anteriormente, para someterlo a discusión y ajuste con el propósito de definir su aplicabilidad y entendimiento. Las afirmaciones positivas o negativas, fueron evaluadas de la siguiente manera: (5) Muy importante, (4) Regularmente importante, (3) Neutral, (2) No tan importante, (1) No es importante en lo absoluto, con respecto al conglomerado al cual pertenecían, de tal manera que la que tenía un mínimo de calificación de $90 \%$ se clasificó como "Buena"; por otra parte, se eliminaron las preguntas con más de $10 \%$ de calificación "Mala".

\section{CAPACITACIÓN DEL PERSONAL}

Los miembros del equipo responsable de la investigación y el trabajo de campo, recibieron capacitación durante 5 días sobre los objetivos, metodología, aplicación de los instrumentos, registro, organización, tabulación y análisis de los datos.

\section{LIMITACIONES}

Se presentaron dificultades para conseguir un segmento de la población de muestra, por el tiempo destinado al estudio y la escasez de algunas formas de donación; la relacionada con donantes remunerados $\mathrm{y}$ altruista.

\section{ANÁLISIS ESTADÍSTICO}

La información se analizó por medio de la creación de una base de datos con la información recolectada y se digitó en Excel, se hizo el análisis bivariado, un análisis básico, primario, ya que la investigación es de tipo descriptivo; esto permitió obtener los datos estadísticos básicos que se conocen, como los porcentajes, frecuencias, probabilidades, prevalencias y razones de prevalencia. Se cálculo la razón de prevalencia con su respectivo intervalo de confianza y la probabilidad estadística, por medio de la prueba de Chi Cuadrado, así mismo, se calcularon las prevalencias de las diferentes categorías de las variables demográficas, con el fin de realizar el correspondiente análisis e interpretación de la información más exhaustiva del proyecto, mostrando el gráfico y la interpretación por cada ítem.
Analizada la información, se vio el impacto que causó esta investigación, para mejorar la participación de la comunidad frente a este acto, y hacer que la donación sea voluntaria y que el Banco de sangre cuente con suficiente reservas para no sufrir necesidades, por lo que el trabajo de investigación se presento ante el personal de Banco de Sangre, del Hospital José María Hernández y al personal del Laboratorio de Salud Publica del Departamento Administrativo de Salud de Putumayo, pues la donación voluntaria forma parte del Plan Territorial de Salud.

\section{RESULTADOS}

Para el trabajo de investigación, se encuestaron 100 personas, quienes respondieron de acuerdo a su saber, logrando así el 100\% del personal de la muestra representativa de la población objeto de estudio. Se tomo en cuenta los siguientes criterios: sexo, nivel de educación, edad, procedencia, sector y estrato socioeconómico. Partiendo de estos criterios, la investigación se realizó en el municipio de Mocoa, con la participación de la población seleccionada (Tabla 2).

Tabla 2. Características de la población seleccionada del municipio de Mocoa - Putumayo, 2008

\begin{tabular}{|c|c|c|}
\hline CARACTERÍSTICA & FRECUENCIA & $\%$ \\
\hline \multicolumn{3}{|c|}{ SEXO } \\
\hline MASCULINO & 49 & 49 \\
\hline FEMENINO & 51 & 51 \\
\hline \multicolumn{3}{|c|}{ NIVEL DE EDUCACIÓN } \\
\hline PRIMARIA & 15 & 15 \\
\hline SECUNDARIA & 41 & 41 \\
\hline TECNOLOGÍA & 13 & 13 \\
\hline UNIVERSITARIA & 22 & 22 \\
\hline PRIMARIA INCOMPLETA & 9 & 9 \\
\hline \multicolumn{3}{|c|}{ EDAD } \\
\hline 18-30 AÑOS & 22 & 22 \\
\hline 31-40 AÑOS & 35 & 35 \\
\hline 41-50 AÑOS & 22 & 22 \\
\hline >50 AÑOS & 21 & 21 \\
\hline \multicolumn{3}{|c|}{ PROCEDENCIA } \\
\hline URBANA & 100 & 100 \\
\hline
\end{tabular}




\begin{tabular}{|c|c|c|}
\hline CARACTERISTICA & FRECUENCIA & $\%$ \\
\hline \multicolumn{3}{|c|}{ SECTOR } \\
\hline ORIENTE & 25 & 25 \\
\hline OCCIDENTE & 25 & 25 \\
\hline NORTE & 25 & 25 \\
\hline SUR & 25 & 25 \\
\hline \multicolumn{3}{|c|}{ ESTRATO SOCIOECONÓMICO } \\
\hline ESTRATO 1 & 50 & 50 \\
\hline ESTRATO 2 & 25 & 25 \\
\hline ESTRATO 3 & 25 & 25 \\
\hline
\end{tabular}

\section{CONOCIMIENTOS DE LA POBLACIÓN}

La población encuestada no ha donado sangre y algunos tienen conocimientos generales sobre la sangre y su transfusión y donación, pero tiene una comprensión relativamente limitada de aspectos específicos (Figura 1).

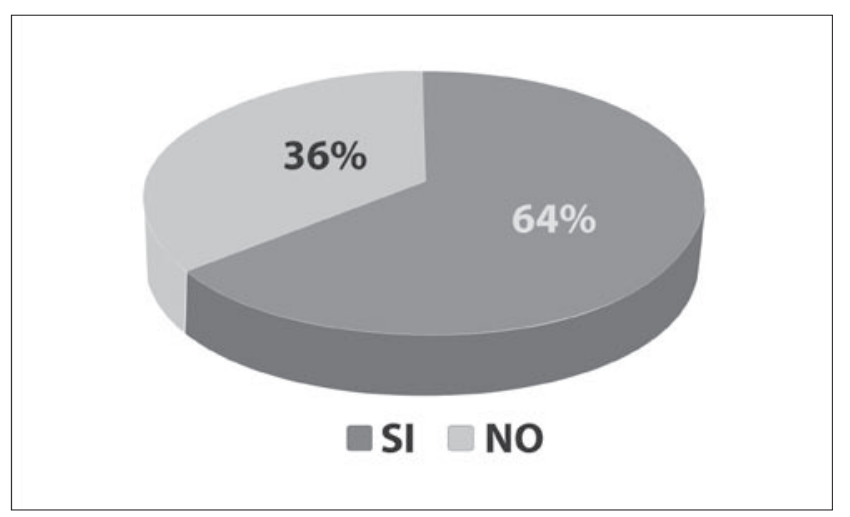

Figura 1. Porcentaje de individuos donantes o no de sangre. Habitantes del municipio de Mocoa - Putumayo, 2008.

La población conoce que la sangre se repone, las funciones de la sangre, algunos conocen su propio tipo de sangre, situaciones más comunes en que una transfusión es necesaria, algunos requisitos y limitaciones para donar sangre, la cantidad de sangre que se extrae y edad mínima para donar (Figura 2).

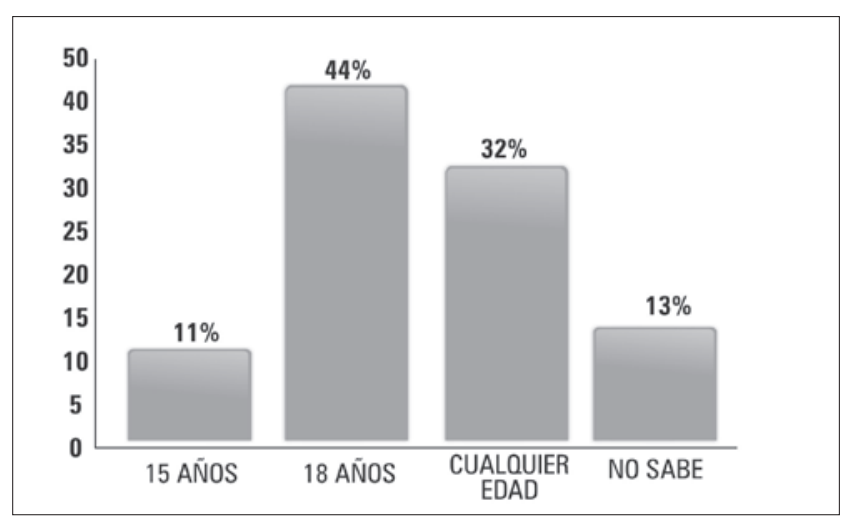

Figura 2. Edad mínima para donar sangre. Habitantes del municipio de Mocoa - Putumayo en 2008.

La población no sabe con claridad el proceso de transfusión, el uso de la sangre donada, la necesidad de sangre, dónde y con qué frecuencia se puede donar, las enfermedades transmisibles y los beneficios y riesgos de donar sangre. Además, en algunas personas persisten mitos sobre la donación de sangre que probablemente influyen negativamente en las prácticas de donación. Algunas son que donar sangre engorda, adelgaza, provoca, disminuye la capacidad sexual entre otras (Figura 3).

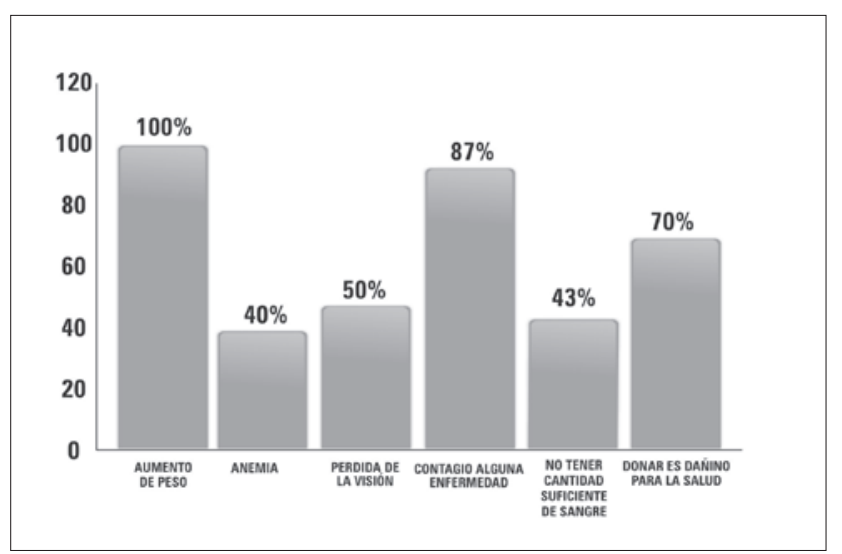

Figura 3. Mitos asociados a la donación de sangre. Habitantes del municipio de Mocoa - Putumayo en 2008.

\section{ACTITUDES}

La mayoría de las personas tienen una actitud positiva, piensan que donar sangre es útil para salvar una vida y algo necesario y saludable. Las personas con actitud negativa, piensan que se puede adquirir alguna enfermedad o aumentar de peso, y le temen al dolor y a la debilidad como consecuencia de la donación. También piensan 
que con la sangre se lucra y se comercializa razón por la cual es difícil conseguir donantes (Figura 4).

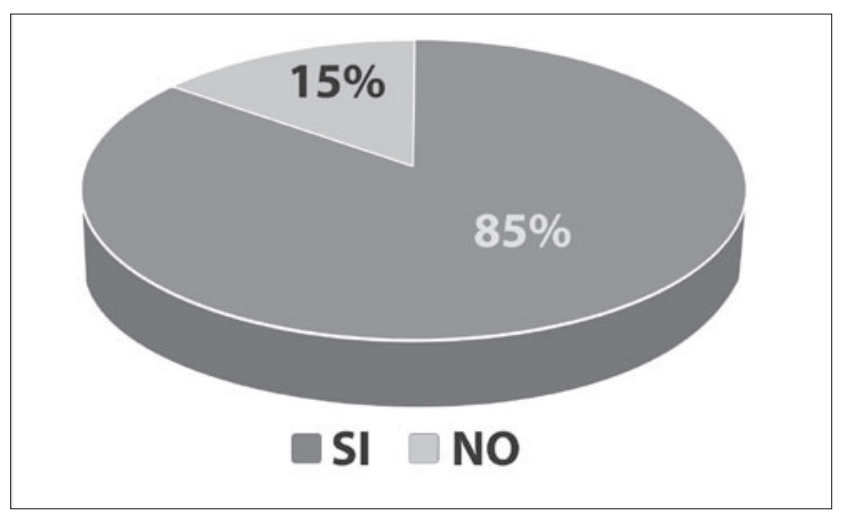

Figura 4. Se comercializa la sangre sí o no. Habitantes del municipio de Mocoa - Putumayo en 2008

En general las personas donarían si lo necesitara un familiar o amigo en caso de urgencia o para su buen estado de salud. Muchos donantes perciben que están donando su sangre para reponer la sangre utilizada por sus familiares; existe poca conciencia de las necesidades permanente de la comunidad. Las personas que nunca han donado, no lo han hecho por falta de oportunidad o por temor a la sangre, a las agujas, al contagio, a debilitarse, o porque creen que no están en condiciones de ser donantes o que no van a recuperar la sangre que donan. Con respecto a los bancos de sangre, la mayor parte de la población, tiene una opinión desfavorable y señalan como elementos de desacierto, el tiempo que toma la donación, la falta de capacitación del personal para la venopunción, la desorganización, la mala atención y los limitantes en infraestructura y equipos.

\section{PRÁCTICAS}

Algunos de los encuestados, hacen cierta práctica de donación, en el Banco de Sangre de la localidad, siempre y cuando sea a un familiar o un amigo cercano; la mayoría donaron su sangre porque alguien se lo pidió y volvería a donar si alguien se lo pidiera. Los voluntarios lo harían para ayudar a cualquier persona que lo necesitara, mientras otras personas donan por interés económico. Esta afirmación se debe a que entre los donantes por reposición, se observa dos actitudes totalmente distintas.

\section{INFORMACIÓN, EDUCACIÓN Y COMUNICACIÓN}

En la actualidad, en el Banco de sangre, realizan actividades de promoción a favor de la donación de sangre, por lo tanto la población ha estado expuesta a mensajes sobre el tema en los diversos medios. No obstante, las personas recomiendan que en el futuro se eduque, informe y motive a la población para que done, por lo que se sugiere tratar temas como los beneficios y riesgos de la donación, despejar los mitos y creencias, e informar sobre el proceso, la necesidad de sangre, la importancia de la donación y el uso de la sangre colectada. Según la población, los mensajes deben ser transmitidos por personal capacitado de La Secretaria de Salud Departamental, Municipal, la Cruz Roja, profesionales de la salud, los propios donantes, líderes de opinión y personas que han sobrevivido gracias a una transfusión, mediante charlas, conferencias, televisión, radio e Internet, impresos o folletos, afiches, prensa, cartillas interactivas, letreros y revistas, mecanismos interpersonales, como cátedras, dramatizaciones y juegos para niños. Es importante utilizar todo el material educativo e informativo al alcance, para la difusión en centros educativos, comunidades, lugares públicos como supermercados, tiendas, parques, servicios de salud, iglesias, lugares de trabajo y los hogares.

\section{DISCUSIÓN}

Cien personas del Municipio de Mocoa, Putumayo, de diferentes puntos cardinales, estratos socioeconómicos y niveles académicos, respondieron satisfactoriamente una encuesta donde se indagó acerca del nivel de conocimiento y la percepción que tenían sobre la donación de sangre.

Los conocimientos sobre la sangre y funciones son generales, algunos brindan una definición específica, pero no es precisa al respecto, igualmente, el conocimiento no está claro sobre cómo y dónde se forma la sangre, ni la cantidad que tenemos en nuestro organismo.

La población encuestada conoce su tipo de sangre. El Grupo predominante en la localidad es el "O", $\mathrm{Rh}$ Positivo, seguido del tipo de sangre "O" negativo $16 \%$, "B" positivo 9\%" A" positivo 8\%, "A" negativo 7\%, "AB" positivo 5\%, "B" negativo 3\% y "AB" negativo $1 \%$ y un $4 \%$ restante no sabe su tipo de sangre.

Los resultados demuestran conocimiento de su tipo y grupo sanguíneo, porque en nuestro país ha sido 
una exigencia para trámites de documentos, más no lo conocen por interés personal o porque hayan participado en el proceso de donación voluntaria, pues el porcentaje de personas que han donado es inferior $(36 \%)$ con relación a quienes no lo han hecho, pues en la actualidad es importante conocer el tipo y grupo de sangre de cada persona, esto ayuda en caso que deba ser transfundida o pueda servir como donante.

Considerando el total de hombres y mujeres que participaron en el estudio, se observó que el 51\% fueron mujeres y un $49 \%$ hombres, quienes tomaron una decisión acertada frente al compromiso de donar, pues se comprobó por medio de un valor de p: 0.15155 y un valor de chi2: 0.6147 , un valor esperado por ser un valor $<5$, con lo que se pudo comprobar que la población quiere colaborar y con su participación se benefician no solo las personas que requieren de este importante líquido, sino también se favorecen los donantes, puesto que estimula y renueva la médula ósea, aumenta la producción de células sanguíneas, disminuye problemas de infarto, oxigena la sangre y permite el diagnostico de alguna enfermedad.

Por otra parte, del personal que participó en el estudio, el $96 \%$ considera necesario donar sangre y un $87 \%$, saben para que se emplea la sangre recolectada por la entidad promotora, además identifican la edad mínima que debe tener una persona para ser donante voluntario o por reposición. Ante esta situación, se observa que hay conocimientos demasiado superficiales al respecto, también se presentan grandes limitantes como las falsas creencias; esto conlleva a asumir una actitud negativa, que se puede corroborar, con las personas que piensan que se puede adquirir alguna enfermedad o aumentar de peso, o les temen al dolor y al decaimiento como consecuencia de la donación ${ }^{5}$.

Si se compara entre los que alguna vez han donado y aquellos que nunca lo han hecho, vemos que el primer grupo tiene conocimientos más precisos, en tanto el segundo grupo lo relaciona más con el hecho de que la sangre es sinónimo de vida, definición que influye en las prácticas de donación, limitando a hacerlo, debido a que si pierde algo de sangre (por donación), pierde algo de vida, percepción cultural arraigada en nuestro país.

La comunidad del municipio de Mocoa, dice en un $65 \%$ que la sangre antes de ser transfundida se somete a análisis, pues el concepto de transfusión no está definido en nuestra población; algunos lo confunden con donación y otros con extracción de sangre. Desconocen el tiempo que debe oscilar entre una donación y otra, es importante tener en cuenta que las donaciones se deben hacer tres veces al año en el caso de las mujeres y los hombres pueden hacerlo 4 veces. Así como los requisitos y limitaciones para hacerlo.

Las personas participantes en el estudio, exponen las razones que influyen en la no donación. En su orden de importancia están: por creer que se comercializa la sangre, la desconfianza en la esterilización de los implementos, falta de información acerca de la donación, muchos atribuyen a causas médicas, temor a la extracción de la sangre, otros tienen mucho temor al ambiente hospitalario, creencias religiosas y en último renglón está la falta de tiempo ${ }^{6}$.

Una de las razones más importantes que desmotivan a la donación sanguínea, es la desconfianza hacia la esterilidad del material, porque piensan que este es reutilizado, no saben con claridad que el material que se utiliza para las donaciones de sangre, es de un solo uso, además es imposible que sea reutilizado ya que tras la donación queda inutilizado para realizar otra extracción, por lo que se genera cierto miedo a contagiarse de alguna enfermedad infecciosa como el SIDA o Hepatitis.

También permite señalar, que la falta de información en la comunidad, desmotiva hacia la participación, como indica los estudios realizados en España, donde se percibe que para los no donantes, la mayor desmotivación es justamente la falta de información. De lo anterior se desprende que deben diseñarse estrategias de comunicación social, eficaces que contemplen información y educación, orientadas a promover la donación voluntaria de sangre.

Del total de la población, en un futuro estarían dispuestos a donar un $86 \%$, los argumentos que los conllevan a colaborar de manera altruista y voluntaria, es en el caso de ayudar a un familiar o amigo enfermo, seguida de una catástrofe nacional. Esto indica que en la comunidad hay bastante solidaridad.

También es una actitud similar a la encontrada en otras personas de América, y una posible explicación sería que en estas regiones se piensa que la sangre es un recurso escaso y por ende, hay que reservar la donación para una situación específica.

La población considera un aspecto muy importante para estar más seguros en su compromiso adquirido, de recibir suficiente capacitación e información por parte de los entes encargados de promocionar la donación, siendo así se logrará mejorar el propósito del Banco de Sangre, otros manifiestan que lo harían cuantas veces sea necesario siempre y cuando reciban una atención apropiada por parte del los profesionales encargados del área. 
Un $70 \%$ de las personas creen que donar es dañino para la salud, porque aumentan de peso, en un 100\%, el pánico de contagiarse de alguna enfermedad, el 87\%, perdida de la visión, y no tener una cantidad suficiente de sangre. Como se puede observar, son motivos sin fundamento que conducen a tomar decisiones equivocadas frente a este tema, es decir, para que las personas no se dispongan a donar sangre.

Estos tabúes y mitos sobre la donación de sangre influyen negativamente en el reclutamiento de donantes voluntarios. Por otra parte, se detectó una leve diferencia entre hombres y mujeres respecto de la actitud positiva hacia una futura donación. A pesar de que esa diferencia no fue estadísticamente significativa, sí concuerda con los resultados obtenidos en estudios, quienes concluyeron que las mujeres están menos dispuestas a donar sangre, en este caso por el miedo que les ocasiona el ambiente hospitalario.

La actitud negativa, está dada por el desconocimiento a lo que es y representa una donación de sangre, así como la desinformación existente, o por la creencia de que uno puede enfermarse o debilitarse. Es importante tomar esto en cuenta, porque constituye una barrera para la donación voluntaria y limita el tener una donación de sangre adecuada para atender casos de emergencia.

Entre los donantes por reposición, se observó dos actitudes totalmente distintas: mientras un grupo lo hace con cierto temor y realiza preguntas frecuentes sobre los procedimientos que le estaban aplicando (donante por reposición), otros se encontraban tranquilos y algunos de ellos estaban pendientes del "familiar" que lo llevó a donar al Banco de Sangre (donante remunerado). Esta apreciación, si bien es cierto es subjetiva; no deja de tener valor al ser hecha por personal capacitado en el manejo de donantes de sangre, con bastantes años de experiencia.

Por tanto, se debe proyectar una perspectiva amplia, con la participación de varios sectores de la población. Los servicios de sangre no tienen la capacidad para adentrarse en los distintos estratos sociales y comunitarios, para llevar la información a todos los ciudadanos y lograr su verdadero compromiso hacia la donación voluntaria de sangre. Para ello es necesario identificar las estructuras existentes en el municipio, en aras de constituir alianzas estratégicas con la participación de distintos actores de los sectores de la salud, la educación y la comunicación.

En resumen, los resultados del presente estudio permiten sugerir que las campañas de promoción de la donación voluntaria de sangre, en la población del Municipio de Mocoa, deberían centrarse en la difusión de información para ampliar conocimientos más precisos sobre los requisitos para ser aceptado como donante, recalcando la seguridad del proceso desde el punto de vista del contagio de enfermedades infecciosas como el SIDA, y la hepatitis entre otras; incorporando una motivación especial para las mujeres, haciendo énfasis en el hecho que, en el proceso de selección de donantes, se realizan una entrevista y un examen físico, para definir si la persona está o no en condiciones de hacer una donación.

Con los resultados obtenidos del estudio, se presenta una serie de sugerencias que pueda contribuir a la ejecución de los cambios necesarios para brindar una mejor calidad de atención al donante y motivarlo a regresar habitualmente, y el diseño de una estrategia de comunicación social para promover la donación de sangre voluntaria en la localidad. Las sugerencias están dirigidas a tres sectores primordiales: la población, el personal de salud y los bancos de sangre.

\section{LA POBLACIÓN}

La mayor motivación enunciada, fue hacer la donación por el hecho de que un familiar o un amigo necesitan sangre, lo que puede llevar a pensar que las personas podrían reservarsudonación para situaciones extremas. Es necesario diseñar una estrategia de comunicación social, que contemple la información, educación y comunicación; concluya tome temas que la población no conoce, y los que desea conocer acerca de la sangre, las transfusiones, la donación, sus experiencias al respecto, actitud hacia la donación, motivaciones para donar, y barreras que limitan la donación ${ }^{7}$.

La estrategia de comunicación, tendrá entonces que dirigirse a despertar la solidaridad como principios básicos en la búsqueda de la seguridad transfusional, con la participación de varios sectores de la población. Los servicios de sangre no tienen la capacidad para adentrarse en los distintos estratos sociales y comunitarios, con el fin de llevar la información a todos los ciudadanos y lograr su compromiso hacia la donación voluntaria de sangre. Para ello es necesario identificar las estructuras existentes en el municipio, con el ánimo de constituir alianzas estratégicas con la participación de los sectores de la salud, la educación y la comunicación directa o personalizada, 
donde se busque un cambio de actitud, con una base fundamentada en establecer programas de educación dirigidos a niños, con miras a sensibilizarlos respecto de la donación de sangre en un futuro.

\section{EL PERSONAL DE SALUD}

La satisfacción del donante, debe ser una preocupación permanente de quienes le prestan el servicio e interactúan con él, por ser un factor determinante en lograr la permanencia de las personas como donantes voluntarios de sangre. El objetivo de los bancos de sangre, no es solo incorporar donantes voluntarios, sino lograr la donación repetida, pues la sangre es más segura cuando proviene de personas mejor informadas y ha sido sometida a pruebas periódicas de laboratorio.

Por tal razón, es importante adoptar medidas y requisitos mínimos, en relación con la selección y atención de los donantes de sangre, tales como el registro antes de la donación, los criterios para la selección de los donantes y su evaluación, las pruebas de tamizaje para la investigación de agentes infecciosos asociados con la transfusión y las normas de bioseguridad.

Se deben implementar capacitaciones de educación continua para el personal del Banco de Sangre y los conocimientos que se requieren para poner en práctica, sobre todo en los temas relacionados con la seguridad de la sangre, componentes, etapas del proceso de donación y atención de cálida al donante, generando un resultado positivo en la mejora del servicio y productividad.

Por último es necesario que, mediante un trabajo de supervisión e inspección constante, se verifique que el personal cumpla con todas las especificaciones técnicas y las recomendaciones de calidad del servicio.

\section{LOS BANCOS DE SANGRE}

La captación a donantes de sangre, está dada por la promoción por parte de la entidad captadora de unidades de sangre y la accesibilidad del servicio y las condiciones bajo las cuales se realiza la donación.

Se recomienda que los bancos de sangre no estén dentro de las instalaciones hospitalarias. Sin embargo, la reorganización del sistema de bancos de sangre, requiere voluntad política y exige una inversión económica alta, por lo que esta decisión solo dependerá del compromiso por parte de las autoridades gubernamentales con un suministro seguro y oportuno de sangre para la población.
Esidealmejorarlainfraestructura, unamejordotaciónde equipos y mobiliario, buscar fuentes de financiamiento y diseñar e implantar un plan de mantenimiento. Además es importante contratar personal suficiente, idóneo y capacitado, con sentido social que se haga cargo de la promoción de donación voluntaria de sangre, reducir el tiempo del procedimiento de donación, ampliar el horario de atención al donante, motivar al donante de reposición e invitarlo a que vuelva a donar su sangre de manera voluntaria. Esto está dentro de la política y el plan nacional de sangre; Se deben contemplar como estrategias para conseguir donantes voluntarios de sangre que sean constantes. Además debe realizar un trabajo educativo con grupos jóvenes, porque en la medida en que se cuente con una población más educada, se podrá lograr que la donación de sangre sea observada como una responsabilidad social del ciudadano.

\section{REFERENCIAS BIBLIOGRÁFICAS}

1. García M, Sáenz de Tejada E, Cruz J. Estudio de factores socioculturales relacionados con la donación voluntaria de sangre en la Américas. Revista Panamericana de Salud Pública. 2003. 1-6

2. Red Solidaria, Información, Donación de sangre. En: Red Solidaria, www.redsolidariacdelu.com. ar/informacion-donante-sangre.php. consulta: noviembre 2008.

3. Alvarez, E. Donar sangre: una prioridad y un deber social. En: Organización Panamericana de la salud, www.col.ops-oms.org/noticias/donarsangre. asp. consulta: noviembre 2008.

4. AvílezJA. Recolección de datos. En: http:// recoldat/ recoldat.shtml. consulta: noviembre 2008.

5. División de Desarrollo de Sistemas y Servicios de Salud. Guía metodológica para investigación de aspectos socio-culturales relacionados con la donación voluntaria de sangre. Washington D.C.: Organización Panamericana de la Salud; 1999. Serie Medicamentos Esenciales y Tecnología, No. 11.

6. Moore RJ. Promoting blood donation: a study of the social profile, attitudes, motivations and experience of donors. Transfusión Medicine. 1991,1:201-207.

7. Rubín G, Carter C, Davies M, et al. Blood Components: A Guide for Patients, 1992. En: http:// www.nhmrc.gov.au/publications/synopses/fiiles/ cp85.pdf 